\title{
Construction of the Magnetic Phase Diagram of FeMn/Ni/Cu(001) Using Photoemission Electron Microscopy
}

\author{
J. $\mathrm{Wu}^{1,2,3}$, A. Scholl ${ }^{4}$, E. Arenholz ${ }^{4}$, Chanyong Hwang ${ }^{5}$, and Z. Q. Qiu ${ }^{3}$ \\ ${ }^{1}$ National High Magnetic Field Laboratory, Florida State University, Tallahassee, FL 32310 USA \\ ${ }^{2}$ Brookhaven National Laboratory, Upton, NY 11973 USA \\ ${ }^{3}$ Department of Physics, University of California Berkeley, Berkeley, CA 94720 USA \\ ${ }^{4}$ Advanced Light Source, Lawrence Berkeley National Laboratory, Berkeley, CA 94720 USA \\ ${ }^{5}$ Division of Advanced Technology, Korea Research Institute of Standards and Science, Yuseong-Gu, Daejeon 305-340, Korea
}

\begin{abstract}
Single crystalline $\mathrm{FeMn} / \mathrm{Ni}$ bilayer was epitaxially grown on $\mathrm{Cu}(001)$ substrate and investigated by photoemission electron microscopy (PEEM). The FeMn and Ni films were grown into two cross wedges to facilitate an independent control of the FeMn (0-20 ML) and Ni (0-20 ML) film thicknesses. The Ni magnetic phases were determined by Ni domain images as a function of the Ni thickness $\left(d_{N i}\right)$ and the FeMn thickness $\left(d_{\mathrm{FeMn}}\right)$. The result shows that as the Ni thickness increases, the Ni film undergoes a paramagnetic-to-ferromagnetic state transition at a critical thickness of $d_{F M}$ and an in-plane to out-of-plane spin reorientation transition at a thicker thickness $d_{S R T}$. The phase diagram shows that both $d_{F M}$ and $d_{S R T}$ increase as the FeMn film establishes its antiferromagnetic order.
\end{abstract}

\section{INTRODUCTION}

A KIN to the semiconductor heterostructures, metallic multilayer system exhibit many fascinating properties that do not exist in single metallic layer system. In particular, magnetic multilayer system integrates spin and charge freedoms of electrons to generate many magneto electronic properties [1]. Among many types of magnetic multilayer systems, antiferromagnetic/ferromagnetic (AFM/FM) systemhas been under intense investigation because of exchange bias effect [2], [3]. The interesting and complexity of the AFM/FM system is at the AFM/FM interfacial magnetic coupling in which the intralayer AFM/AFM and FM/FM interactions compete with the interfacial coupling to generate the so-called spin frustration. Since spin frustration occurs at atomic scale, surface roughness plays an important role in the AFM/FM interaction. In an effort to minimize the interfacial roughness, single crystalline $\mathrm{FeMn} / \mathrm{Co} / \mathrm{Cu}(001)$ thin films have become one of the model systems for the study of interfacial interactions in AFM/FM system [4]. Previous studies show that the AFM order of the FeMn layer significantly affect the Co magnetic properties [5], [6]. The observed phenomena were well explained by the $\mathrm{FeMn} / \mathrm{Co}$ interaction due to the presence of random atomic steps at the interface. Further studies on vicinal surfaces isolate different effects of the atomic steps along different crystal orientations [7]. Encouraged by the success of epitaxial AFM/FM layered systems, it is highly demanded to develop other systems to explore the effect of the AFM layer on various magnetic properties of the FM layer. In a FM system, the two most important properties are the magnetic order and the magnetization orientation. The former is determined by the magnetic exchange interaction, and the latter is determined by the magnetic anisotropy. We recently showed that the AFM order of FeMn could change the perpendicular anisotropy of a Ni layer in $\mathrm{FeMn} / \mathrm{Ni} / \mathrm{Cu}(001)$ system [8]. To reveal systematically how the AFM layer affect these two properties, we carried out a study on epitaxial $\mathrm{FeMn} / \mathrm{Ni} / \mathrm{Cu}(001)$ system as a function of FeMn and Ni film thicknesses. Using photoemission electron spectroscopy (PEEM), we studied the $\mathrm{Ni}$ paramagnetic-to-ferromagnetic phase transition and the in-pane to out-of-plane spin reorientation transition (SRT). We find that the antiferromagnetic order of the FeMn layer decreases the $\mathrm{Ni}$ Curie temperature and increases the Ni SRT thickness.

A $10 \mathrm{~mm}$ diameter $\mathrm{Cu}(001)$ substrate is prepared by electric polishing and cycles of sputtering at $2 \mathrm{KeV}$ and annealing at $600 \mathrm{C}$. Low energy electron diffraction (LEED) and auger electron spectroscopy (AES) were used to characterize the substrate to ensure the formation of a clean $\mathrm{Cu}(001)$ surface. $\mathrm{Ni}$ and $\mathrm{Fe}^{50} \mathrm{Mn}^{50}$ films were grown at room temperature by evaporating $\mathrm{Ni}$ and co-evaporating $\mathrm{Fe}$ and $\mathrm{Mn}$ from alumina crucibles. The Ni (0-20 ML) and $\mathrm{Fe}^{50} \mathrm{Mn}^{50}(0-20 \mathrm{ML})$ films were grown into cross-wedged shapes by translating the substrate behind a knife-edge shutter in two orthogonal directions during the film deposition. The slope of the wedge is fully determined by the moving speed of the substrate motion and the film deposition rate. In this way, the wedged sample cover a thickness range continuously in a single sample. Since the wedged film was grown under the same growth condition, the variation due to different sample preparation conditions can be eliminated to ensure a systematic thickness dependent study. Therefore the double wedged $\mathrm{FeMn} / \mathrm{Ni}$ sample enables a systematic study of the magnetic phase diagram as a function of both $\mathrm{Ni}$ and $\mathrm{FeMn}$ thicknesses. The sample is covered by a $2 \mathrm{~nm} \mathrm{Cu}$ protection layerbefore being taken from our growth vacuum chamber to the photoemission electron microscopy (PEEM) station of Advanced Light Source of Lawrence Berkeley National Laboratory. In the PEEM measurement, the X-ray is circularly polarized and the photon energy is tuned to the resonant absorption L2 and L3 peaks of the Ni. Photoelectrons are collected to generate element-specific magnetic domain contrast utilizing the effect of X-ray magnetic circular dichroism (XMCD). Typical 


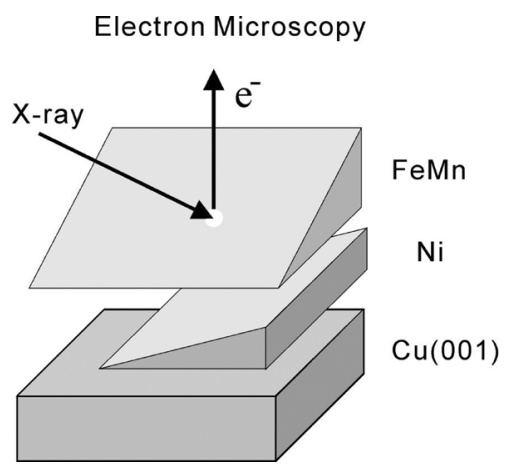

Fig. 1. Schematic drawing of the sample and PEEM measurement geometry. The incident X-ray is circularly polarized and is at a $60^{\circ}$ incident angle from the sample surface normal direction.

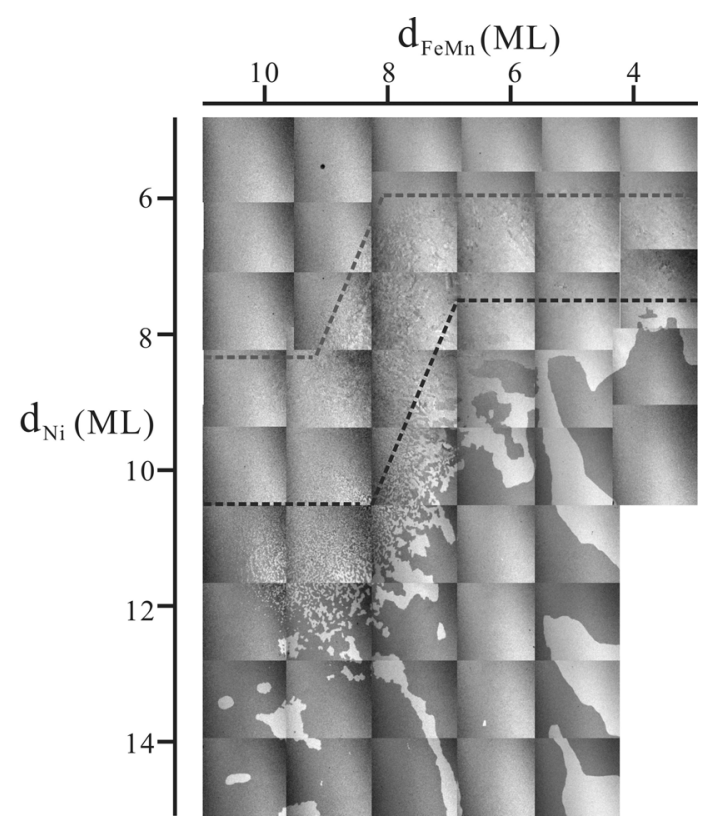

Fig. 2. Room temperature PEEM images of the Ni magnetic domains at different Ni and FeMn thicknesses. At each FeMn thickness, the Ni undergoes a paramagnetic to in-plane ferromagnetic transition and an in-plane to out-ofplane spin reorientation transition as the Ni thicknesses increases.

magnetic spatial resolution of PEEM image is about $100 \mathrm{~nm}$. By taking PEEM images across our double wedge, we can obtain the Ni magnetic phase diagram with a high accuracy in the $\mathrm{Ni}$ and FeMn thickness plane. In the PEEM measurement, the $\mathrm{X}$-ray was incident at $60^{\circ}$ from the surface normal direction (Fig. 1) so that both in-plane and out-of-plane magnetization components can be picked up by the PEEM.

Fig. 2 shows Ni magnetic domain images as a function of both $\mathrm{Ni}$ and FeMn thicknesses. The first observation of Fig. 2 is that the FeMn/Ni bilayer behaves differently for thin and thick FeMn, e.g., the Ni domain undergoes a transition in the region of $7<\mathrm{d}_{\mathrm{FeMn}}<9 \mathrm{ML}$. Recall that FeMn film at room temperature undergoes the paramagnetic-to-antiferromagnetic transition at $d_{\mathrm{FeMn}} \sim 7-9 \mathrm{ML}$ [6], the different Ni domain patterns in Fig. 2 must reflect the effects of FeMn at paramagnetic state at $\mathrm{d}_{\mathrm{FeMn}}<7-9 \mathrm{ML}$ and at antiferromagnetic state at $\mathrm{d}_{\mathrm{FeMn}}>7-9 \mathrm{ML}$.
We first discuss the Ni domains at the paramagnetic region of FeMn. As the Ni thickness increases, there are three different characteristic Ni magnetic states. Below 6 ML thickness, the Ni film exhibits no magnetic domains. By changing the X-ray from left to right circular polarization, we find no difference in the $\mathrm{Ni}$ absorption spectrum at the L2 and L3 peaks. This result shows that the Ni below $6 \mathrm{ML}$ at room temperature is at the paramagnetic state rather than being at the single domain state, i.e., the Curie temperature of the Ni film is below room temperature for $\mathrm{d}_{\mathrm{Ni}}<6 \mathrm{ML}$, in agreement with the literature result [9]. For $\mathrm{d}_{\mathrm{Ni}}>6 \mathrm{ML}$, the Ni film exhibits clearly magnetic domains, showing that the Ni film is at the ferromagnetic state. Therefore $\mathrm{d}_{\mathrm{Ni}}=6 \mathrm{ML}$ is the critical thickness of which the Ni film undergoes the paramagnetic-to-ferromagnetic phase transition at room temperature, i.e., a $6 \mathrm{ML} \mathrm{Ni}$ film has its Curie temperature equal to the room temperature. Even in the ferromagnetic state, we notice different types of Ni magnetic domains in different thickness regions. For $6 \mathrm{ML}<\mathrm{d}_{\mathrm{Ni}}<7.5 \mathrm{ML}$, the $\mathrm{Ni}$ film processes small sized scattered shape domain pattern. By rotating the sample 90 degrees around the surface normal direction, we find that the domain contrast changes with some dark color domains changing into white color domains. Notice that the PEEM domain contrast is from the XMCD effect which is proportional to the projection of X-ray incident direction to the local Ni spin orientation, the change of domain contrast after rotating the sample 90 degrees around the surface normal direction shows that the Ni spin is in the film plane for $6 \mathrm{ML}<\mathrm{d}_{\mathrm{Ni}}<7.5$ ML. Above $\mathrm{d}_{\mathrm{Ni}}>7.5 \mathrm{ML}$, the Ni domain size becomes relatively larger and only has two color contrast (black/white). After rotating the sample by 90 degrees around the sample surface normal direction, we find that the $\mathrm{Ni}$ domain contrast remains unchanged, showing that the Ni spin is in the out-of-plane direction at $\mathrm{d}_{\mathrm{Ni}}>7.5 \mathrm{ML}$. Thus, $\mathrm{d}_{\mathrm{Ni}}=7.5 \mathrm{ML}$ corresponds to the critical thickness that the Ni film undergoes a spin reorientation transition (SRT) from in-plane below 7.5 ML to out-of-plane direction above 7.5 ML. This kind of SRT has been observed in $\mathrm{Ni} / \mathrm{Cu}(001)$ system [10]. Here we show that the SRT also occurs after the $\mathrm{Ni} / \mathrm{Cu}(001)$ is covered with FeMn layer.

Next, we discuss the $\mathrm{Ni}$ domains at the antiferromagnetic region of $\mathrm{FeMn}$ in $\mathrm{FeMn} / \mathrm{Ni} / \mathrm{Cu}(001)$. Three magnetic phases were identified as a function of the Ni thickness, corresponding to the paramagnetic phase, in-plane ferromagnetic phase, and out-of-plane ferromagnetic phase. These three phases are the same as in the $\mathrm{FeMn} / \mathrm{Ni} / \mathrm{Cu}(001)$ as the FeMnlayer is at paramagnetic state. However, the critical Ni thicknesses for the paramagnetic-to-ferromagnetic transition and for the SRT are different as compared to the paramagnetic state of FeMn.

We first discuss the Ni paramagnetic-to-ferromagnetic phase transition in FeMn/Ni for antiferromagnetic phase of FeMn. The $\mathrm{Ni}$ critical thickness of this transition $\mathrm{d}_{\mathrm{FM}}$ is $8.5 \mathrm{ML}$ for antiferromagnetic FeMn overlayer as opposed to the 6.0 ML for paramagnetic FeMn overlayer. Note that the Curie temperature of a ferromagnetic thin film increases with increasing film thickness [11], the thicker critical Ni thickness of 8.5 ML than 6.0 ML shows that the antiferromagnetic order of FeMn overlayerlowers the Curie temperature of Ni layer, i.e., the interfacial coupling between the ferromagnetic $\mathrm{Ni}$ and the antiferromagnetic FeMn lowers the Curie temperature of the Ni film. This 
result is different from the case of the interfacial coupling between two ferromagnetic layers of which the interfacial coupling should always increases the Curie temperature of the films [12]. The microscopic mechanism is that the FM ordered film can polarize the adjacent interfacial layerwhich is equivalent to an increase of the ferromagnetic layer thickness, leading to a higher Curie temperature. For the case of $\mathrm{FeMn} / \mathrm{Ni}$ bilayer, we observe the exact opposite result. Here we offer a hand waving explanation. For the FM/FM bilayer case, the interfacial coupling is equivalent of applying an effective magnetic field from one FM layer to the other thus helping the establishment of the FM order of the second layer. For the AFM/FM bilayer case, the interfacial coupling occurs between the FM spins which are parallel to each other and the AFM spins which are alternating their directions between nearest neighbors [for compensated spin surface such as the FeMn(001) surface]. Therefore, the AFM order is equivalent of applying a local magnetic field, which alternates its direction between nearest neighbors, to the FM layer at the interface. This "random" magnetic field obviously has an effect of destroying the parallel alignment of the FM layer spins to promote a loss of the FM order. This explains why the AFM order of the FM layer decreases the Curie temperature of the $\mathrm{Ni}$ layer (or increases the $\mathrm{Ni}$ critical thickness) in $\mathrm{FeMn} / \mathrm{Ni}$ bilayer system. But we should point out that a loss of macroscopic magnetization of the Ni film does not necessarily mean the loss of magnetic order. Random field could establish a spin glass magnetic order in the Ni film which also leads to a loss of macroscopic magnetization. A final distinguish between paramagnetic and spinglass states needs a susceptibility measurement at the transition point.

We then discuss the Ni SRT in FeMn/Ni for the antiferromagnetic phase transition of FeMnoverlayer. First, in the region where FeMn changes from paramagnetic to antiferromagnetic $\left(7<\mathrm{d}_{\mathrm{FeMn}}<9 \mathrm{ML}\right)$ and Ni SRT takes place $\left(\mathrm{d}_{\mathrm{Ni}}=10.5\right.$ $\mathrm{ML}$ ), the out-of-plane $\mathrm{Ni}$ domains breaks into relatively small domains. This phenomenon is similar to the domain change in $\mathrm{Co} / \mathrm{FeMn}$ system, which is attributed to the establishment of the FeMnantiferromagnetic order [5]. This is an important signature of the FeMn AFM order because metallic FeMn does not generate X-ray Magnetic Linear Dichroism so that there is no effective way to measure the FeMn AFM order. Then dramatic result is that the Ni SRT critical thickness is $10.5 \mathrm{ML}$ for AFM ordered FeMn which is $40 \%$ higher than the $7.5 \mathrm{ML}$ of the Ni SRT critical thickness for paramagnetic FeMn. Generally speaking, the spin direction of a FM thin film is determined by its magnetic anisotropy. The SRT of Ni film in Ni/Cu(001) is a result of the competition among the shape anisotropy, surface anisotropy, and the volume anisotropy [13]. Since all these three anisotropies should be stabilized within a few atomic layers of the FeMnoverlayer, the change of the Ni SRT shows that the AFM order of the FeMnoverlayer introduces an additional magnetic anisotropy to the $\mathrm{Ni}$ film.

To understand why the FeMn AFM order induces a magnetic anisotropy, we notice that the magnetic interfacial interaction between FeMn and Ni is tuned on only after the establishment of the FeMn AFM order. The magnetic energy of the Ni layer can be expressed in terms of its spin components which correspond to the magnetic anisotropy terms. Thus, the effect of

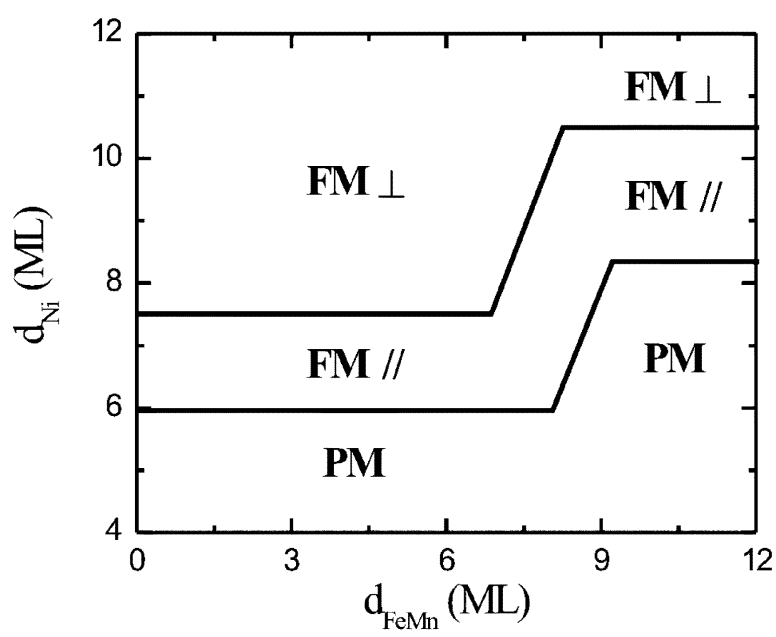

Fig. 3. Ni magnetic phase diagram derived from the PEEM images in Fig. 2. $\mathrm{PM}, \mathrm{FM} / /$, and $\mathrm{FM} \perp$ indicate paramagnetic, in-plane ferromagnetic, and out-of-plane ferromagnetic phases.

the $\mathrm{FeMn} / \mathrm{Ni}$ interfacial interaction should also be expressed as an anisotropy term. That is why the Ni layer in the $\mathrm{FeMn} / \mathrm{Ni}$ system experiences different magnetic anisotropies at paramagnetic and antiferromagnetic states of the FeMn film. To further understand the microscopic mechanism, one needs to consider the spin structure of FeMn. For FeMn (001) atomic planes, the nonlinear 3Q-like spin structure [14] has a zero net spin for the in-plane spin component but a non-zero out-of-plane spin component which alternates its direction between neighboring (001) planes. Then at the FeMn/Ni interface with the presence of atomic steps (inevitable in real experimental systems), the nonzero perpendicular spin component at each atomic terrace interacts with the Ni layer. Then the presence of atomic steps will lead to a magnetic frustration [15] that the FeMn-Ni interfacial interaction favors an alternating alignment of the $\mathrm{Ni}$ spins between neighboring terraces in the surface normal direction and that the Ni-Ni ferromagnetic interaction prefers a parallel alignment of the Ni spins. This kind of magnetic frustration usually ends up a 90 degree spin alignment between the AFM and FM spin orientations by introducing an effective uniaxial magnetic anisotropy to the FM layer which favors the FM spin being perpendicular to the AFM spins [16]. Another way to understand the AFM/FM 90-degree coupling is that the effect of the FM to the AFM layer in the AFM/FM interaction is equivalent of applying a magnetic field (FM layer magnetization) to the AFM layer. Then the well-known "spin-flop" state in antiferromagnets [17] results in the switching of the AFM spins to the perpendicular direction of the magnetic field. For the AFM/FM bilayer case, the "spin-flop" transition corresponds to a switching of the FM spins to the perpendicular direction of the AFM spins. Then for the specific case of $\mathrm{FeMn} / \mathrm{Ni} / \mathrm{Cu}(001)$ system, the $\mathrm{FeMn} / \mathrm{Ni}$ out-of-plane interfacial spin frustration should generate a magnetic anisotropy that favors the Ni spins to be perpendicular to the film normal direction, i.e., favors the $\mathrm{Ni}$ spins to be in the plane of the film. That is why the AFM order of the FeMnoverlayershifts the Ni SRT thickness to a thicker thickness by changing the $\mathrm{Ni}$ magnetization direction in the region of 7.5 ML $<\mathrm{d}_{\mathrm{Ni}}<10.5 \mathrm{ML}$ from out-of-plane direction 
at paramagnetic FeMn state to in-plane direction at antiferromagnetic state of FeMn.

Based on the magnetic domain images, the Ni magnetic phase diagram at room temperature is established and shown in Fig. 3. The two solid lines mark out the phase boundaries between the paramagnetic phase, in-plane ferromagnetic phase, and out-ofplane ferromagnetic phase.

In summary, we fabricated $\mathrm{FeMn} / \mathrm{Ni}$ bilayer into cross wedges on $\mathrm{Cu}(001)$ and employed PEEM to construct a complete Ni magnetic phase diagram at room temperature. Three magnetic phases are identified and the phase boundaries are determined. The different value of the $\mathrm{Ni}$ critical thicknesses for the two phase transitions is attributed to the paramagnetic and antiferromagnetic phases of the FeMnoverlayer. We conclude that the antiferromagnetic order of the FeMn at $d_{F e M n}>7-9$ ML increases the Ni critical thickness for both transitions, indicating that the FeMn antiferromagnetic order decreases the Curie temperature of the $\mathrm{Ni}$ film and also induces an in-plane magnetic anisotropy to the Ni film.

\section{ACKNOWLEDGMENT}

This work was supported by National Science Foundation DMR-0803305, U.S. Department of Energy DE-AC0205CH11231, ICQS of Chinese Academy of Science, and KICOS through Global Research Laboratory project.

\section{REFERENCES}

[1] S. D. Bader and S. S. P. Parkin, Ann. Rev. Condens. Matter Phys., vol. 1, pp. 71-88, 2010.

[2] W. H. Meiklejohn and C. P. Bean, Phys. Rev., vol. 102, p. 1413, 1956.

[3] J. Nogues and I. K. Schuller, J. Magn. Magn. Mater., vol. 192, no. 203 , 1999.

[4] W. Kuch, L. I. Chelaru, F. Offi, J. Wang, M. Kotsugi, and J. Kirschner, Phys. Rev. Lett., vol. 92, p. 017201, 2004.

[5] W. Kuch, F. Offi, L. I. Chelaru, M. Kotsugi, K. Fukumoto, and J. Kirschner, Phys. Rev. B, vol. 65, p. 140408, 2002.

[6] C. Won, Y. Z. Wu, H. W. Zhao, A. Scholl, A. Doran, W. Kim, T. L. Owens, X. F. Jin, and Z. Q. Qiu, Phys. Rev. B, vol. 71, p. 024406, 2005.

[7] J. Choi, J. Wu, Y. Z. Wu, C. Won, A. Scholl, A. Doran, T. Owens, and Z. Q. Qiu, Phys. Rev. B, vol. 76, p. 054407, 2007.

[8] J. Wu, J. Choi, A. Scholl, A. Doran, E. Arenholz, C. Hwang, and Z. Q. Qiu, Phys. Rev. B, vol. 79, p. 212411, 2009.

[9] M. Farle, B. Mirwald-Schulz, A. N. Anisimov, W. Platow, and K. Baberschke, Phys. Rev. B, vol. 55, p. 3708, 1997.

[10] B. Schulz and K. Baberschke, Phys. Rev. B, vol. 50, p. 13467, 1994.

[11] R. Zhang and R. F. Willis, Phys. Rev. Lett., vol. 86, p. 2665, 2001.

[12] C. Won, Y. Z. Wu, A. Scholl, A. Doran, N. Kurahashi, H. W. Zhao, and Z. Q. Qiu, Phys. Rev. Lett., vol. 91, p. 147202, 2003.

[13] M. Farle, W. Platow, A. N. Anisimov, P. Poulopoulos, and K. Baberschke, Phys. Rev. B, vol. 56, p. 5100, 1997.

[14] T. C. Schulthess, W. H. Butler, G. M. Stocks, S. Maat, and G. J. Mankey, J. Appl. Phys., vol. 85, p. 4842, 1999.

[15] U. Schlickum, N. Janke-Gilman, W. Wulfhekel, and J. Kirschner, Phys. Rev. Lett., vol. 92, p. 107203, 2004.

[16] N. C. Koon, Phys. Rev. Lett., vol. 78, p. 4865, 1997.

[17] R. Jungblut, R. Coehoorn, M. Johnson, J. aan de Stegge, and A. Reinders, J. Appl. Phys., vol. 75, p. 6659, 1994. 
This document was prepared as an account of work sponsored by the United States Government. While this document is believed to contain correct information, neither the United States Government nor any agency thereof, nor the Regents of the University of California, nor any of their employees, makes any warranty, express or implied, or assumes any legal responsibility for the accuracy, completeness, or usefulness of any information, apparatus, product, or process disclosed, or represents that its use would not infringe privately owned rights. Reference herein to any specific commercial product, process, or service by its trade name, trademark, manufacturer, or otherwise, does not necessarily constitute or imply its endorsement, recommendation, or favoring by the United States Government or any agency thereof, or the Regents of the University of California. The views and opinions of authors expressed herein do not necessarily state or reflect those of the United States Government or any agency thereof or the Regents of the University of California. 